\title{
Special issue on genetic fuzzy systems: new advances
}

\author{
Rafael Alcalá $\cdot$ Yusuke Nojima
}

Published online: 23 October 2009

(C) Springer-Verlag 2009

After almost forty years of development, fuzzy systems have demonstrated their superb ability to solve different problems arising in various application domains. In the last two decades, the cooperative framework established made the research interest move to augment fuzzy systems with learning and adaptation capabilities. Since the first pioneer works dated back to 1991 [1-4], one of the most successful approaches to hybridize fuzzy systems with learning and adaptation methods, apart of course from fuzzy neural networks, has resulted in the so-called Genetic Fuzzy Systems (GFSs) [5-9]. These hybrid computational intelligence techniques augment the approximate reasoning method of fuzzy systems with the learning capabilities of evolutionary algorithms.

This topic has attracted considerable attention in the Computational Intelligence community in the last few years $[9,10]$. Contrary to neural networks, clustering, rule induction and many other machine learning approaches, Genetic Algorithms (GAs) provide a means to encode and evolve rule antecedent aggregation operators, different rule semantics, rule base aggregation operators and defuzzification methods. Therefore, GAs remain today as one of the fewest knowledge acquisition schemes available to design

R. Alcalá ( $₫)$

Department of Computer Science and A.I.,

University of Granada, 18071 Granada, Spain

e-mail: alcala@decsai.ugr.es

URL: http://www.ugr.es/ ralcala/

\section{Y. Nojima}

Department of Computer Science and Intelligent Systems,

Osaka Prefecture University, 1-1 Gakuen-cho, Naka-ku,

Sakai, Osaka 599-8531, Japan

e-mail: nojima@cs.osakafu-u.ac.jp

URL: http://www.cs.osakafu-u.ac.jp/ nojima/ and, in some sense, optimize fuzzy systems with respect to the design decisions, allowing decision makers to decide what components are fixed and which ones evolve according to the performance measures.

The predominant type of GFS is that focused on fuzzy rule-based systems. However other kinds of GFSs have been developed, with successful results. Thanks to the high potential of GFSs, we observe the increase of published papers in the topic in the last few years. A non-exhaustive list of recent journal papers on Genetic Fuzzy Rule-Based Systems can be found at [11], as a part of the Website "Genetic Fuzzy Systems: Taxonomy, Current Research Trends and Prospects" [9, 10]. Paying attention to the specialized literature we can find two authored books [5, 6] and three edited ones [12-14], together with a list of journal special issues devoted to GFSs [15-22], including important contributions to all branches of GFSs.

Further, a Series of Workshops on the topic has been established to support GFSs. The series started in Granada, Spain, with the First International Workshop on Genetic Fuzzy Systems (GFS2005) [23] celebrated between the 17th and the 19th of March, 2005, went on with the 2006 International Symposium on Evolving Fuzzy Systems (EFS2006) [24] held in Lake District, UK, between the 7th and the 9th of September, 2006, and with the Third International Workshop on Genetic and Evolving Fuzzy Systems (GEFS2008) [25] held in Witten-Bommerholz, Germany, between the 4th and the 7th of March, 2008, and will continue with the Fourth International Workshop on Genetic and Evolutionary Fuzzy Systems (GEFS2010) [26] to be organized next March, 17-19, 2010 in Mieres, Spain.

Taking into account all these facts, it can be recognized how the GFS research area has reached its maturity and how many special topics have sound roots and have been largely developed. This Special Issue pays now attention to 
recent and significant research efforts on GFSs in order to deal with current challenges on this topic. To this end, the five papers in this special issue address distinct subjects focusing on new, significant novel lines of development on GFSs.

In the first one, entitled "Evolutionary Parallel and Gradually Distributed Lateral Tuning of Fuzzy Rule-Based Systems”, I. Robles, R. Alcalá, J. M. Benítez and F. Herrera deal with a novel research line that is becoming important as a way to address large scale problems, the use of parallel distributed GFSs. Distributed genetic algorithms are excellent optimization algorithms which exploit the nowadays available parallel hardware (multi-core microprocessors and clusters) and could help to alleviate the complex search space of these kinds of problems. In this contribution, the authors present a study on the use of the distributed genetic algorithms for the tuning of Mamdani fuzzy rule-based systems. To this end, they analyze the application of a specific gradual distributed real-coded genetic algorithm which employs eight subpopulations in a hypercube topology and local parallelization at each subpopulation. The proposal is evaluated in nine real-world data sets of different sizes and with different numbers of variables, using non-parametric statistical tests for pairwise comparisons. The results shows that the distributed approach achieves better results in terms of quality and execution time as the complexity of the problem grows.

The paper "Multi-objective Evolutionary Learning of Granularity, Membership Function Parameters and Rules of Mamdani Fuzzy Systems" by M. Antonelli, P. Ducange, B. Lazzerini and F. Marcelloni focuses on a current and very important research trend, the use of multi-objective evolutionary algorithms to improve the interpretabilityaccuracy trade-off of fuzzy rule-based systems. This paper presents a specific multi-objective evolutionary algorithm to learn the rule base and the data base (granularities and membership function parameters) of Mamdani fuzzy rulebased systems. It is a new derivation approach considering the following two objectives: minimization of the number of conditions used in the antecedents of the rules and minimization of the mean square error. To this aim, the authors introduce the concept of virtual and concrete partitions: the former is defined by uniformly partitioning each linguistic variable with a fixed maximum number of fuzzy sets; the latter takes into account, for each variable, the number of fuzzy sets determined by the evolutionary process. Rule bases and membership function parameters are defined on the virtual partitions and, whenever a fitness evaluation is required, mapped to the concrete partitions by employing appropriate mapping strategies. The proposed algorithm is tested on three real-world regression problems achieving very promising results with good trade-offs between complexity and accuracy.
Another contribution in the same topic is that by A. A. Márquez, F. A. Márquez and A. Peregrín, entitled "Rule Base and Adaptive Fuzzy Operators Cooperative Learning of Mamdani Fuzzy Systems with Multi-objective Genetic Algorithms", which is also a significant proposal on the improvement of the interpretability-accuracy trade-off of Mamdani fuzzy rule-based systems by using multi-objective evolutionary algorithms. The authors present a new multi-objective evolutionary approach to achieve cooperation between the rule base and the adaptive fuzzy operators of the inference system in order to obtain simpler, more compact and still accurate linguistic fuzzy models by learning fuzzy inference adaptive operators together with the rule base. It is an approach and not just an algorithm, because there is not only one algorithm, but several combinations of "stages". All of these combinations performs a data-driven learning seeking to find good values for the parameters of the adaptable conjunction and defuzzification operators considered as well as selecting rules from a wider set of rules consistent with the data. The multiobjective nature of the algorithms is justified for the two opposite objectives considered: minimization of the number of rules and minimization of the mean square error. The search algorithms are based on SPEA2 and NSGA-II, whose evolutions are hinted through the use of the socalled 'Guided Domination Approach'. The performance of this proposal is evaluated through a experimental setup which considers nine real-world regression problems and by applying non-parametric statistical tests for pair-wise comparisons. The results confirm the usefulness of the proposed approach.

The paper "Multiagent Coevolutionary Genetic Fuzzy System to Develop Bidding Strategies in Electricity Markets: Computational Economics to Assess Mechanism Design" by I. Walter and F. Gomide is related to the design of new learning schemes on new real-world application areas. In this contribution, the authors suggest a co-evolutionary genetic fuzzy system to develop bidding strategies for agents in online auction environments. Particularly, they study how the co-evolutionary fuzzy bidding strategies perform against each other during hourly electric energy auctions. The authors show how co-evolutionary approaches developed by co-evolutionary agents interact through their fuzzy bidding strategies in a multiagent environment, allowing more realistic and transparent representations of agents behavior in auction-based markets. Further, they show that this way working also improves market representation and evaluation mechanisms. The paper deals with a hot application domain topic. The use of a competitive co-evolutionary genetic fuzzy system seems to be a very sensible, sound and accurate solution procedure when it is applied on actual public data available from a Brazilian independent system operator. 
Finally, A. M. Palacios, L. Sánchez and I. Couso focus on a significantly innovative GFS proposal in the last contribution to the issue entitled "Extending a Simple Genetic Cooperative-Competitive Learning Fuzzy Classifier to low quality datasets". Exploiting the information in low quality data sets has been recently acknowledged as a new challenge in genetic fuzzy systems. Within this framework, this paper presents a new proposal to adapt genetic fuzzy classification systems to deal with vague data from low quality data sets, i.e., to deal with the necessity for managing intervals, fuzzy data and missing attributes. In order to do that, this last contribution proposes some modifications on a simple cooperative-competitive learning fuzzy classifier: the inference system, the fitness, and the ranking of such fitness values. The proposed algorithm becomes the first example of a genetic fuzzy classifier which is able to use low quality data. The authors demonstrate the good performance of the proposed approach by using a synthetic benchmark and two real-world problems. These two real-world problems constitute themselves new real-world application areas for the use of GFSs.

Acknowledgments The Guest Editors would like to thank the contributors who answered our call and the reviewers for their careful work in making this special issue possible. On the other hand, they would also like to express their gratitude to the Evolutionary Intelligence Editor-in-Chief, Larry Bull, for providing us with the opportunity to edit this issue and for his kind help in the editorial process.

\section{References}

1. Karr C (1991) Genetic algorithms for fuzzy controllers. AI Expert 6(2):26-33

2. Pham DT, Karaboga D (1991) Optimum design of fuzzy logic controllers using genetic algorithms. J Syst Eng 1:114-118

3. Thrift P (1991) Fuzzy logic synthesis with genetic algorithms. In: Proceedings of fourth international conference on genetic algorithms (ICGA'91). Morgan Kaufmann, San Diego, pp 509-513

4. Valenzuela-Rendón M (1991) The fuzzy classifier system: motivations and first results. In: Schwefel HP, Männer R (eds) Proceedings of first international conference on parallel problem solving from nature-PPSN I. Springer, Berlin, pp 330-334
5. Geyer-Schulz A (1995) Fuzzy rule-based expert systems and genetic machine learning. Physica-Verlag, Heidelberg

6. Cordón O, Herrera F, Hoffmann F, Magdalena L (2001) Genetic fuzzy systems. Evolutionary tuning and learning of fuzzy knowledge bases. World Scientific, Singapore

7. Cordón O, Gomide F, Herrera F, Hoffmann F, Magdalena L (2004) Ten years of genetic fuzzy systems: current framework and new trends. Fuzzy Sets Syst 141(1):5-31

8. Herrera F (2005) Genetic fuzzy systems: status, critical considerations and future directions. Int J Comput Intell Res 1(1):59-67

9. Herrera F (2008) Genetic fuzzy systems: taxonomy, current research trends and prospects. Evol Intell 1:27-46

10. http://www.sci2s.ugr.es/gfs/

11. http://www.sci2s.ugr.es/gfs/biblio.php

12. Herrera F, Verdegay JL (eds) (1996) Genetic algorithms and soft computing. Physica-Verlag, Heidelberg

13. Sanchez E, Shibata, Zadeh L (eds) (1997) Genetic algorithms and fuzzy logic systems. Soft computing perspectives, World Scientific, Singapore

14. Pedrycz W (ed) (1997) Fuzzy evolutionary computation. Kluwer, Norwell

15. Herrera F (1997) Special issue on genetic fuzzy systems for control and robotics. Int J Approx Reason 17(4):325-326

16. Herrera F, Magdalena L (1998) Special issue on genetic fuzzy systems. Int J Intell Syst 13(10-11):887-890

17. Cordón O, Herrera F, Hoffmann F, Magdalena L (2001) Special issue on recent advances in genetic fuzzy systems. Inf Sci 136(14): $1-5$

18. Cordón O, Gomide F, Herrera F, Hoffmann F, Magdalena L (2004) Special issue on genetic fuzzy systems. Fuzzy Sets Syst 141(1): $1-3$

19. Casillas J, del Jesus MJ, Herrera F, Prez R, Villar P (2007) Special issue on genetic fuzzy systems and the interpretabilityaccuracy trade-off. Int J Approx Reason 44(1):1-3

20. Cordón O, Alcalá R, Alcalá-Fdez J, Rojas I (2007) Special issue on genetic fuzzy systems: what's next? IEEE Trans Fuzzy Syst 15(4):533-535

21. Carse B, Pipe AG (2007) Special issue on genetic fuzzy systems. Int J Intell Syst 22(9):905-907

22. Casillas J, Carse B (2009) Special issue on genetic fuzzy systems: recent developments and future directions. Soft Comput 13(5):417-418

23. http://www.sci2s.ugr.es/gfs2005

24. http://www.efs06.org

25. http://www.gefs08.de

26. http://www.softcomputing.es/gefs 2010 